\title{
Suitability of Microbes for Bio-modification of Geomaterial during MICP Process
}

\author{
Surabhi Jain ${ }^{1}$, Dali Naidu Arnepalli ${ }^{1}$ \\ ${ }^{1}$ Indian Institute of Technology Madras \\ Department of Civil Engineering, Chennai-600036, India \\ ce14d062@smail.iitm.ac.in; arnepalli@iitm.ac.in
}

\section{Extended Abstract}

Microbially induced calcium carbonate precipitation (MICP) is a sustainable biocementation process in which the carbonate-based minerals are precipitated within the voids and at the interface of the particulate assembly, thereby altering the engineering behaviour of the porous media [1]. The successful implementation of the MICP process, for improving the geotechnical properties of coarse-grained soils, encounters numerous challenges such as soil conditions (soil $\mathrm{pH}$, geometric compatibility), environmental condition (oxygen level, cementation reagents concentration, temperature), biotic condition (type and concentration of biomass) etc. [2,3].

In view of this, the present study dedicated for assessing the suitability of various ureolytic microbes viz. two strains of Sporosarcina pasteurii (denoted as BP1, BP2) and Proteus species (denoted as P1) for improving the shear strength behaviour of poorly graded coarse-grained sand, over a range of environmental conditions. For this purpose, a series of sand columns were prepared and 1.5 pore volume of biomass was imbibed, later it was allowed for curing of 12 hours, to enable the adherence of microbes on the surface of the sand particles. Subsequently, 1.5 pore volume of an equimolar concentration of cementation solution (a mixture of $0.25 \mathrm{M}$ urea, $0.5 \mathrm{M}$ calcium chloride and minimal nutrient broth) was injected into the sand column at every 12 hours interval for 10 days. Control specimens were also prepared by treating them under identical conditions as above but no microbial activity.

The bio-modified sand has shown variable amounts of mineral precipitation, and enhancement of unconfined compressive strength (UCS). The variation in UCS is observed to be in the order of 0.8 to $1.1 \mathrm{MPa}$ when the sand is treated with microbes BP2 and P1 using cementation solution of 0.25 to $0.5 \mathrm{M}$ concentration, respectively. On the contrary, the sand treated with microbe BP1 under similar chemical, biotic and environment has not resulted in measurable mineral precipitation and caused an unnoticeable change in UCS. The amount and type of polymorph of the biomineral formed, which in turn expected to govern the engineering properties of the bio-modified sand, is also varied with the type of microorganism employed as well as the concentration of the cementation agent. In order to ascertain the reason for the above-mentioned observations, bench-scale experiments were also conducted in an Erlenmeyer flask by employing all the microbes considered in this study under aerobic, anoxic (air restricted) and anaerobic environmental conditions. The results indicated no biomineral precipitation by the microbe BP1 when treated in an anaerobic environment, and the amount of biomineral precipitated is meager in an anoxic environment. The meager or no precipitation of biomineral is due to the hindrance of microbial growth in these environments. The observed variation in the quantity of biomineral formed in aerobic environments by the three organisms is primarily ascribed to variations in their urease enzyme activity in these environments. Overall, the study concluded that the growth rate and enzymatic activity of various organisms considered in this study are predominantly influenced by the prevailing environmental conditions, which in turn determines the efficiency of MICP process in modifying the engineering properties of soil.

\section{References}

[1] D. Mujah, M. A. Shahin, and L. Cheng, "State-of-the-art review of bio-cementation by microbially induced calcite precipitation (MICP) for soil stabilization," Geomicrobiol. J., vol. 34, no. 6, pp. 524-537, 2016.

[2] B. M. Mortensen, M. J. Haber, J. T. Dejong, L. F. Caslake, and D. C. Nelson, "Effects of environmental factors on microbial induced calcium carbonate precipitation," J. Appl. Microbiol., vol. 111, no. 2, pp. 338-349, 2011. 
[3] W. Ng, M. Lee, and S. Hii, "An overview of the factors affecting microbial-induced calcite precipitation and its potential application in soil improvement," World Acad. Sci. Eng. Technol., vol. 62, no. 2, pp. 723-729, 2012. 